\title{
Existence of Multiple Solutions for Fourth-Order Elliptic Problem
}

\author{
Hua Gu and Tianqing An \\ College of Science, Hohai University, Nanjing 210098, China \\ Correspondence should be addressed to Hua Gu; guhuasy@hhu.edu.cn
}

Received 11 April 2014; Accepted 11 August 2014; Published 28 August 2014

Academic Editor: Felix Sadyrbaev

Copyright (C) 2014 H. Gu and T. An. This is an open access article distributed under the Creative Commons Attribution License, which permits unrestricted use, distribution, and reproduction in any medium, provided the original work is properly cited.

By using the variant fountain theorem, we study the existence of multiple solutions for a class of superquadratic fourth-order elliptic problem with Navier boundary value condition.

\section{Introduction}

Consider the following fourth-order boundary value problem:

$$
\begin{gathered}
\Delta^{2} u+c \Delta u=g(x, u) \quad \text { in } \Omega, \\
u=\Delta u=0 \quad \text { on } \partial \Omega,
\end{gathered}
$$

where $\Delta^{2}$ denotes the biharmonic operator, $\Omega \subset \mathbb{R}^{N}(N>4)$ is a bounded domain with smooth boundary, and $g \in C(\bar{\Omega} \times$ $\mathbb{R}, \mathbb{R})$.

The fourth-order elliptic equations which contain a biharmonic operator can describe the static form change of beam or the motion of rigid body. Thus the fourth-order elliptic equations are widely applied in physics, oceanics, aerospace engineering and other engineering. In [1], Lazer and Mckenna considered the biharmonic problem:

$$
\begin{gathered}
\Delta^{2} u+c \Delta u=d\left[(u+1)^{+}-1\right] \quad \text { in } \Omega, \\
u=\Delta u=0 \quad \text { on } \partial \Omega,
\end{gathered}
$$

where $u^{+}=\max \{u, 0\}$ and $d \in \mathbb{R}$. They pointed out that this type of nonlinearity furnishes a model to study traveling waves in suspension bridges. Afterwards, in [2], they have proved the existence of $2 k-1$ solutions when $N=1$ and $d>\lambda_{i}\left(\lambda_{i}-c\right)\left(\left\{\lambda_{i}\right\}_{i \geq 1}\right.$ is the sequence of the eigenvalues of $-\Delta$ in $\left.H_{0}^{1}(\Omega)\right)$ by the global bifurcation method. In [3] the existence of a negative solution of (2) was proved when $d>\lambda_{1}\left(\lambda_{1}-c\right)$ by using the Leray-Schauder degree. In particular, in $[1,4]$ the authors observed that problem (2) was interesting also when the nonlinearity $(u+1)^{+}-1$ was replaced by a somewhat more general function $g(\cdot, u)$. In [5], Micheletti and Pistoia used a variational linking theorem to investigate the existence of two solutions for a more general nonlinearity $g(\cdot, u)$. Moreover, by using a variational result, they and Saccon also showed the existence of three solutions for some special $g(\cdot, u)$ (see [6]). Next year, in [7], Micheletti and Saccon obtained two results about the existence of two nontrivial solutions and four nontrivial solutions by the similar variational approach, depending on the position of a suitable parameter with respect to the eigenvalues of the linear part. In recent years, more researchers have used variational approach to investigate the fourth-order elliptic equations. In [8], Xu and Zhang studied the existence of positive solutions of problem (1) when $g$ satisfied the local superlinearity and sublinearity condition and $c<\lambda_{1}$ by the classical mountain pass theorem. Recently, in [9], Pu et al. used the least action principle, the Ekeland variational principle, and the mountain pass theorem to prove the existence and multiplicity of solutions of (1) when $g(x, u)=$ $a(x)|u|^{s-2} u+f(x, u)\left(a \in L^{\infty}(\Omega), s \in(1,2)\right)$. For other related results, see [8-14] and the references therein. Here, we emphasize that most authors considered the case $c<\lambda_{1}$.

The variant fountain theorems established in [15] have been used in the study of a class of semilinear elliptic equations (see $[16,17]$ ) and the investigation of the Hamiltonian 
system (see $[18,19])$. Inspired by $[9,17]$, we will use the variant fountain theorem to investigate the problem (1). More precisely, we make the following assumptions.

$\left(S_{1}\right)$ There exist constants $d_{1}>0$ and $1<v<(N+4) /(N-$ 4) such that

$$
|g(x, u)| \leq d_{1}\left(1+|u|^{\nu}\right), \quad \forall(x, u) \in \Omega \times \mathbb{R} .
$$

$\left(S_{2}\right) G(x, u) \geq 0$ for all $(x, u) \in \Omega \times \mathbb{R}$ and

$$
\liminf _{|u| \rightarrow \infty} \frac{G(x, u)}{|u|^{2}}=\infty, \quad \text { uniformly for } x \in \Omega \text {. }
$$

Here, $G(x, u):=\int_{0}^{u} g(x, s) d s$ is the primitive of the nonlinearity $g$.

$\left(S_{3}\right)$ There exist constants $\varrho>(2 N /(N+4)) \nu, L>0$ and $d_{3}>0$ such that

$$
u g(x, u)-2 G(x, u) \geq d_{3}|u|^{\varrho} \quad \forall|u| \geq L, x \in \Omega .
$$

Our main result is the following theorem.

Theorem 1. Assume that $\left(S_{1}\right)-\left(S_{3}\right)$ hold and $G(x, u)$ is even in $u$. Then problem (1) possesses infinitely many solutions.

Remark 2. In Theorem 1, we do not assume $c<\lambda_{1}$, which is widely used in the investigation of the fourth-order equations. As is known, the so-called global AmbrosettiRabinowitz condition (AR-condition for short) is introduced by Ambrosetti and Rabinowitz in [20] and wildly used to the existence of infinitely many solutions for superquadratic situation: there is a constant $\alpha>2$ such that, for all $u \neq 0$ and $x \in \Omega$, the nonlinearity is assumed to satisfy

$$
0<\alpha G(x, u) \leq u g(x, u)
$$

In fact, if we choose

$$
G(x, u)=H(x)\left(|u|^{\mu}+(\mu-2)|u|^{\mu-\varepsilon} \sin ^{2}\left(\frac{|u|^{\varepsilon}}{\varepsilon}\right)\right)
$$

where $\varepsilon \in(0, \mu-2), H \in C(\bar{\Omega})$, and $H(x)>0$ for all $x \in \bar{\Omega}$. Then it is easy to see that $G$ satisfies the conditions $\left(S_{1}\right)-\left(S_{3}\right)$ in Theorem 1 with $\mu=3, v=2, \varepsilon=0.1, \varrho=2.9$, and $N=5$, but $G$ does not satisfy the AR-condition (6).

Remark 3. By $\left(S_{1}\right)$, we can obtain that there exists a constant $d_{2}>0$ such that

$$
|G(x, u)| \leq d_{1}\left(|u|+|u|^{\nu+1}\right)+d_{2}, \quad \forall(x, u) \in \Omega \times \mathbb{R} .
$$

And by $\left(S_{3}\right)$, there exists a constant $d_{4}>0$ such that

$$
u g(x, u)-2 G(x, u) \geq d_{3}|u|^{\varrho}-d_{4}, \quad \forall(x, u) \in \Omega \times \mathbb{R}
$$

\section{Preliminaries}

In this section, we will establish the variational setting for our problem and state a variant fountain theorem.

Let $E=H^{2}(\Omega) \cap H_{0}^{1}(\Omega)$ be the Hilbert space equipped with the inner product

$$
(u, v)_{E}=\int_{\Omega} \Delta u \Delta v d x
$$

and the norm

$$
\|u\|_{E}=(u, v)_{E}^{1 / 2}
$$

A weak solution of problem (1) is a $u \in E$ such that

$$
\int_{\Omega}(\Delta u \Delta v-c\langle\nabla u, \nabla v\rangle) d x-\int_{\Omega} g(x, u) v d x=0
$$

for any $v \in E$. Here and in the sequel, $\langle\cdot, \cdot\rangle$ always denotes the standard inner product in $\mathbb{R}^{N}$. Let $\Phi: E \rightarrow R$ be the functional defined by

$$
\Phi(u)=\frac{1}{2} \int_{\Omega}\left(|\Delta u|^{2}-c|\nabla u|^{2}\right) d x-\int_{\Omega} G(x, u) d x .
$$

It is well known that a critical point of the functional $\Phi$ in $E$ corresponds to a weak solution of problem (1).

Let $\lambda_{i}(i=1,2, \ldots)$ be the eigenvalues of $-\Delta$ in $H_{0}^{1}(\Omega)$. Then the eigenvalue problem

$$
\begin{gathered}
\Delta^{2} u+c \Delta u=\mu u \quad \text { in } \Omega, \\
u=\Delta u=0 \quad \text { on } \partial \Omega,
\end{gathered}
$$

has infinitely many eigenvalues $\mu_{i}=\lambda_{i}\left(\lambda_{i}-c\right), i=1,2, \ldots$ by

Define a selfadjoint linear operator $\mathscr{A}: L^{2}(\Omega) \rightarrow L^{2}(\Omega)$

$$
(\mathscr{A} u, v)_{2}=\int_{\Omega}(\Delta u \Delta v-c\langle\nabla u, \nabla v\rangle) d x
$$

with domain $D(\mathscr{A})=E$. Here, $(\cdot, \cdot)_{2}$ denotes the inner product in $L^{2}(\Omega)$ and in the sequel $L^{2}(\Omega)$ is simply denoted by $L^{2}$. Then the sequence of eigenvalues of $\mathscr{A}$ is just $\left\{\mu_{i}\right\}$ (i= $1,2, \ldots)$. Denote the corresponding system of eigenfunctions by $\left\{e_{n}\right\}$; it forms an orthogonal basis in $L^{2}$.

Denote

$$
n^{-}=\#\left\{i \mid \mu_{i}<0\right\}, \quad n^{0}=\#\left\{i \mid \mu_{i}=0\right\}, \quad \bar{n}=n^{-}+n^{0} .
$$

Here, $\#\{\cdot\}$ denotes the cardinal of a set. Let

$$
\begin{gathered}
L^{-}=\operatorname{span}\left\{e_{1}, \ldots, e_{n^{-}}\right\}, \quad L^{0}=\operatorname{span}\left\{e_{n^{-}+1}, \ldots, e_{\bar{n}}\right\}, \\
L^{+}=\left(L^{-} \oplus L^{0}\right)^{\perp}=\overline{\operatorname{span}\left\{e_{\bar{n}+1}, \ldots,\right\} .}
\end{gathered}
$$

Decompose $L^{2}$ as

$$
L^{2}=L^{-} \oplus L^{0} \oplus L^{+}
$$


Then $E$ also possesses the orthogonal decomposition

$$
E=E^{-} \oplus E^{0} \oplus E^{+}
$$

with

$$
E^{-}=L^{-}, \quad E^{0}=L^{0}, \quad E^{+}=E \cap L^{+}=\overline{\operatorname{span}\left\{e_{\bar{n}+1}, \ldots,\right\}} .
$$

We define on $E$ a new inner product and the associated norm by

$$
\begin{gathered}
(u, v)=\left(\mathscr{A} u^{+}, v^{+}\right)_{2}-\left(\mathscr{A} u^{-}, v^{-}\right)_{2}+\left(u^{0}, v^{0}\right)_{2} \\
\|u\|=(u, u)^{1 / 2}
\end{gathered}
$$

Therefore, $\Phi$ can be written as

$$
\Phi(u)=\frac{1}{2}\left(\left\|u^{+}\right\|^{2}-\left\|u^{-}\right\|^{2}\right)-\Psi(u),
$$

where $\Psi(u)=\int_{\Omega} G(x, u) d x$ for all $u=u^{-}+u^{0}+u^{+} \in E=$ $E^{-} \oplus E^{0} \oplus E^{+}$. Then $\Phi$ and $\Psi$ are continuously differentiable.

Direct computation shows that

$$
\begin{gathered}
\Psi^{\prime}(u) v=\int_{\Omega} g(x, u) v d x \\
\Phi^{\prime}(u) v=\left(u^{+}, v^{+}\right)-\left(u^{-}, v^{-}\right)-\Psi^{\prime}(u) v
\end{gathered}
$$

for all $u, v \in E$ with $u=u^{-}+u^{0}+u^{+}$and $v=v^{-}+v^{0}+v^{+}$, respectively. It is known that $\Psi^{\prime}: E \rightarrow E$ is compact.

Denote by $\|\cdot\|_{p}$ the usual norm of $L^{p} \equiv L^{p}(\Omega)$ for all $1 \leq$ $p \leq 2 N /(N-4)$; then by the Sobolev embedding theorem, there exists a $\tau_{p}>0$ such that

$$
\|u\|_{p} \leq \tau_{p}\|u\|, \quad \forall u \in E
$$

Noting that the constants $\nu$ and $\varrho$ appeared in $\left(S_{1}\right)$ and $\left(S_{3}\right)$ satisfies

$$
1+v<\frac{2 N}{N-4}, \quad \frac{\varrho}{\varrho-v}<\frac{2 N}{N-4} .
$$

To prove our main result Theorem 1, we need an abstract critical point theorem found in [15].

Let $E$ be a Banach space with the norm $\|\cdot\|$ and $E=\overline{\oplus_{j \in \mathbb{N}} X_{j}}$ with $\operatorname{dim} X_{j}<\infty$ for any $j \in \mathbb{N}$. Set $Y_{k}=\oplus_{j=1}^{k} X_{j}$ and $Z_{k}=$ $\overline{\oplus_{j=k}^{\infty} X_{j}}$. Consider the following $C^{1}$-functional $\Phi_{\lambda}: E \rightarrow \mathbb{R}$ defined by

$$
\Phi_{\lambda}(u):=A(u)-\lambda B(u), \quad \lambda \in[1,2] .
$$

Theorem 4 (see [15, Theorem 2.1]). Assume that the functional $\Phi_{\lambda}$ defined above satisfies the following:

$\left(F_{1}\right) \Phi_{\lambda}$ maps bounded sets to bounded sets for $\lambda \in[1,2]$, and $\Phi_{\lambda}(-u)=\Phi_{\lambda}(u)$ for all $(\lambda, u) \in[1,2] \times E$;

$\left(F_{2}\right) B(u) \geq 0$ for all $u \in E$; moreover, $A(u) \rightarrow \infty$ or $B(u) \rightarrow \infty$ as $\|u\| \rightarrow \infty$
$\left(F_{3}\right)$ there exist $r_{k}>\rho_{k}>0$ such that

$$
\alpha_{k}(\lambda):=\inf _{u \in Z_{k},\|u\|=\rho_{k}} \Phi_{\lambda}(u)>\beta_{k}(\lambda):=\max _{u \in Y_{k},\|u\|=r_{k}} \Phi_{\lambda}(u),
$$

$\forall \lambda \in[1,2]$.

Then

$$
\alpha_{k}(\lambda) \leq \zeta_{k}(\lambda):=\inf _{\gamma \in \Gamma_{k}} \max _{u \in B_{k}} \Phi_{\lambda}(\gamma(u)), \quad \forall \lambda \in[1,2],
$$

where $B_{k}=\left\{u \in Y_{k}:\|u\| \leq r_{k}\right\}$ and $\Gamma_{k}:=\left\{\gamma \in C\left(B_{k}, E\right)\right.$ : $\gamma$ is odd, $\left.\left.\gamma\right|_{\partial B_{k}}=i d\right\}$. Moreover, for a.e. $\lambda \in[1,2]$, there exists a sequence $\left\{u_{m}^{k}(\lambda)\right\}_{m=1}^{\infty}$ such that

$$
\begin{gathered}
\sup _{m}\left\|u_{m}^{k}(\lambda)\right\|<\infty, \quad \Phi_{\lambda}^{\prime}\left(u_{m}^{k}(\lambda)\right) \longrightarrow 0, \\
\Phi_{\lambda}\left(u_{m}^{k}(\lambda)\right) \longrightarrow \zeta_{k}(\lambda) \\
\text { as } m \longrightarrow \infty .
\end{gathered}
$$

In order to apply this theorem to prove our main result, we define the functionals $A, B$, and $\Phi_{\lambda}$ on our working space $E=H^{2}(\Omega) \cap H_{0}^{1}(\Omega)$ as follows:

$$
\begin{aligned}
A(u)= & \frac{1}{2}\left\|u^{+}\right\|^{2}, \quad B(u)=\frac{1}{2}\left\|u^{-}\right\|^{2}+\int_{\Omega} G(x, u) d x \\
\Phi_{\lambda}(u) & =A(u)-\lambda B(u) \\
& =\frac{1}{2}\left\|u^{+}\right\|^{2}-\lambda\left(\frac{1}{2}\left\|u^{-}\right\|^{2}+\int_{0}^{T} G(x, u) d x\right)
\end{aligned}
$$

for all $u=u^{-}+u^{0}+u^{+} \in E=E^{-}+E^{0}+E^{+}$and $\lambda \in[1,2]$. Then $\Phi_{\lambda} \in C^{1}(E, \mathbb{R})$ for all $\lambda \in[1,2]$ and

$$
\Phi_{\lambda}^{\prime}(u) v=\left(u^{+}, v^{+}\right)-\lambda\left(\left(u^{-}, v^{-}\right)+\int_{\Omega} g(x, u) v d x\right) .
$$

Let $X_{j}=\operatorname{span}\left\{e_{j}\right\}, j=1,2, \ldots$ Note that $\Phi_{1}$ is just equal to the functional $\Phi$ defined in (22).

\section{Proof of Theorem 1}

In this section we firstly establish the following two lemmas and then give the proof of Theorem 1 .

Lemma 5. Assume that $\left(S_{1}\right)$ and $\left(S_{2}\right)$ hold. Then $B(u) \geq 0$ for all $u \in E$. Furthermore, $A(u) \rightarrow \infty$ or $B(u) \rightarrow \infty$ as $\|u\| \rightarrow \infty$.

Proof. Since $G(x, u) \geq 0$, by (30), it is obvious that $B(u) \geq 0$ for all $u \in E$.

By the similar method used in the proof of Lemma 2.6 of [17], for any finite-dimensional subspace $F \subset E$, there exists a constant $\epsilon>0$ such that

$$
m(\{x \in \Omega:|u| \geq \epsilon\|u\|\}) \geq \epsilon, \quad \forall u \in F \backslash\{0\},
$$

where $m(\cdot)$ is the Lebesgue measure in $\mathbb{R}^{N}$. 
Now for the finite-dimensional subspace $E^{-} \oplus E^{0} \subset E$, there exists a constant $\epsilon$ corresponding to the one in (33). Let

$$
\Lambda_{u}=\{x \in \Omega:|u| \geq \epsilon\|u\|\}, \quad \forall u \in E^{-} \oplus E^{0} \backslash\{0\} .
$$

Then $m\left(\Lambda_{u}\right) \geq \epsilon$. By $\left(S_{2}\right)$, there exist positive constants $d_{5}$ and $R_{1}$ such that

$$
G(x, u) \geq d_{5}|u|^{2}, \quad \forall x \in \Omega,|u| \geq R_{1} .
$$

Note that

$$
|u(x)| \geq R_{1}, \quad \forall x \in \Lambda_{u}
$$

for any $u \in E^{-} \oplus E^{0}$ with $\|u\| \geq R_{1} / \epsilon$. Combining (35) and (36), for any $u \in E^{-} \oplus E^{0}$ with $\|u\| \geq R_{1} / \epsilon$, we have

$$
\begin{aligned}
B(u) & =\frac{1}{2}\left\|u^{-}\right\|^{2}+\int_{\Omega} G(x, u) d t \\
& \geq \int_{\Lambda_{u}} G(x, u) d t \geq \int_{\Lambda_{u}} d_{5}|u|^{2} d t \\
& \geq d_{5} \epsilon^{2}\|u\|^{2} \cdot m\left(\Lambda_{u}\right) \geq d_{5} \epsilon^{3}\|u\|^{2},
\end{aligned}
$$

which implies that

$$
B(u) \longrightarrow \infty \text { as }\|u\| \longrightarrow \infty \text { on } E^{-} \oplus E^{0} .
$$

Combining this with $E=E^{-} \oplus E^{0} \oplus E^{+}$and (30), we have

$$
A(u) \longrightarrow \infty \quad \text { or } \quad B(u) \longrightarrow \infty \quad \text { as }\|u\| \longrightarrow \infty .
$$

The proof is completed.

Lemma 6. Let $\left(S_{1}\right),\left(S_{2}\right)$ be satisfied. Then there exist a positive integer $k_{1}$ and two sequences $r_{k}>\rho_{k} \rightarrow \infty$ as $k \rightarrow \infty$ such that

$$
\begin{gathered}
\alpha_{k}(\lambda):=\inf _{u \in Z_{k},\|u\|=\rho_{k}} \Phi_{\lambda}(u)>0, \quad \forall k \geq k_{1}, \\
\beta_{k}(\lambda):=\max _{u \in Y_{k},\|u\|=r_{k}} \Phi_{\lambda}(u)<0, \quad \forall k \in \mathbb{N},
\end{gathered}
$$

where $Y_{k}=\oplus_{j=1}^{k} X_{j}=\operatorname{span}\left\{e_{1}, e_{2}, \ldots, e_{k}\right\}$ and $Z_{k}=\overline{\oplus_{j=k}^{\infty} X_{j}}=$ $\overline{\operatorname{span}\left\{e_{k}, e_{k+1}, \ldots\right\}}$ for all $k \in \mathbb{N}$.

Proof.

Step 1. We first prove (40).

By virtue of (8) and (31), for any $u \in E^{+}$

$$
\begin{aligned}
\Phi_{\lambda}(u) \geq \frac{1}{2}\|u\|^{2}-2 \int_{\Omega} G(x, u) d x & \\
\geq \frac{1}{2}\|u\|^{2}-2 d_{1}\left(\|u\|_{1}+\|u\|_{\nu+1}^{\nu+1}\right)- & 2 d_{2} \cdot m(\Omega), \\
& \forall \lambda \in[1,2],
\end{aligned}
$$

where $d_{1}, d_{2}$ are the constants in (8). Let

$$
\iota_{\nu+1}(k)=\sup _{u \in Z_{k},\|u\|=1}\|u\|_{\nu+1}, \quad \forall k \in \mathbb{N} .
$$

Then

$$
\iota_{\nu+1}(k) \longrightarrow 0 \quad \text { as } k \longrightarrow \infty
$$

since $E$ is compactly embedded into $L^{\nu+1}$. Note that

$$
Z_{k} \subset E^{+}, \quad \forall k \geq \bar{n}+1,
$$

where $\bar{n}$ is the integer given in (16). Combining (24), (42), (43), and (45), for $k \geq \bar{n}+1$, we have

$$
\begin{aligned}
\Phi_{\lambda}(u) \geq & \frac{1}{2}\|u\|^{2}-2 d_{1} \tau_{1}\|u\|-2 d_{2} \cdot m(\Omega) \\
& -2 d_{1} \iota_{\nu+1}^{\nu+1}(k)\|u\|^{\nu+1}, \quad \forall(\lambda, u) \in[1,2] \times Z_{k},
\end{aligned}
$$

where $\tau_{1}$ is the constant given in (24). By (44), there exists a positive integer $k_{1} \geq \bar{n}+1$ such that

$$
\begin{aligned}
\rho_{k}:= & \left(16 d_{1} \iota_{\nu+1}^{\nu+1}(k)\right)^{1 /(1-\nu)} \\
& >\max \left\{16 d_{1} \tau_{1}+1,16 d_{2} \cdot m(\Omega)\right\}, \quad \forall k \geq k_{1}
\end{aligned}
$$

since $v>1$. Clearly,

$$
\rho_{k} \longrightarrow \infty \quad \text { as } k \longrightarrow \infty
$$

Combining (46) and (47), direct computation shows

$$
\alpha_{k}(\lambda):=\inf _{u \in Z_{k},\|u\|=\rho_{k}} \Phi_{\lambda}(u) \geq \frac{\rho_{k}^{2}}{4}>0, \quad \forall k \geq k_{1} .
$$

Step 2. We then prove (41).

Note that for any $k \in \mathbb{N}, Y_{k}$ is of finite dimension, so we can choose $M_{1}>0$ sufficiently large such that

$$
\|u\| \leq M_{1}\left(\int_{\Omega}|u|^{2}\right)^{1 / 2}, \quad \forall u \in Y_{k} .
$$

By $\left(S_{2}\right)$ and (8), for the former $M_{1}$, there exists a $M_{2}>0$ such that

$$
G(x, u) \geq M_{1}^{2}|u|^{2}-M_{2}, \quad \forall(t, u) \in[0, T] \times \mathbb{R}^{N} .
$$

Consequently, by (50) and (51), we have

$\Phi_{\lambda}(u)$

$$
\begin{aligned}
\leq & \frac{1}{2}\left\|u^{+}\right\|^{2}-\frac{1}{2}\left\|u^{-}\right\|^{2}-\int_{\Omega} G(x, u) d t \\
\leq & \frac{1}{2}\left\|u^{+}\right\|^{2}-\frac{1}{2}\left\|u^{-}\right\|^{2}-M_{1}^{2} \int_{\Omega}|u|^{2} d t \\
& +M_{2} \cdot m(\Omega) \\
\leq & \frac{1}{2}\left\|u^{+}\right\|^{2}-\frac{1}{2}\left\|u^{-}\right\|^{2} \\
& -M_{1}^{2}\left(\frac{1}{M_{1}^{2}}\left\|u^{+}\right\|^{2}+\frac{1}{M_{1}^{2}}\left\|u^{0}\right\|^{2}\right)+M_{2} \cdot m(\Omega)
\end{aligned}
$$

$\leq-\frac{1}{2}\left\|u^{+}\right\|^{2}-\frac{1}{2}\left\|u^{-}\right\|^{2}-\left\|u^{0}\right\|^{2}+M_{2} \cdot m(\Omega)$

$\leq-\frac{1}{2}\|u\|^{2}+M_{2} \cdot m(\Omega)$ 
for all $u=u^{-}+u^{0}+u^{+} \in Y_{k}$. Now for any $k \in \mathbb{N}$, if we choose

$$
r_{k}>\max \left\{\rho_{k}, \sqrt{2 M_{2} \cdot m(\Omega)}\right\}
$$

then (52) implies

$$
\beta_{k}(\lambda):=\max _{u \in Y_{k},\|u\|=r_{k}} \Phi_{\lambda}(u)<0, \quad \forall k \in \mathbb{N} .
$$

The proof is completed.

Now we prove our main result Theorem 1.

Proof of Theorem 1. In view of (8), (24), and (31), $\Phi_{\lambda}$ maps bounded sets to bounded sets uniformly for $\lambda \in[1,2]$. By virtue of the evenness of $G(x, u)$ in $u$, it holds that $\Phi_{\lambda}(-u)=$ $\Phi_{\lambda}(u)$ for all $(\lambda, u) \in[1,2] \times E$. Therefore the condition $\left(F_{1}\right)$ of Theorem 4 holds. Lemma 5 shows that the condition $\left(F_{2}\right)$ holds, whereas Lemma 6 implies that condition $\left(F_{3}\right)$ holds for all $k \geq k_{1}$, where $k_{1}$ is given in Lemma 6 . Thus, by Theorem 4 , for each $k \geq k_{1}$ and a.e. $\lambda \in[1,2]$, there exists a sequence $\left\{u_{m}^{k}(\lambda)\right\}_{m=1}^{\infty} \subset E$ such tha

$$
\begin{gathered}
\sup _{m}\left\|u_{m}^{k}(\lambda)\right\|<\infty, \quad \Phi_{\lambda}^{\prime}\left(u_{m}^{k}(\lambda)\right) \longrightarrow 0, \\
\Phi_{\lambda}\left(u_{m}^{k}(\lambda)\right) \longrightarrow \zeta_{k}(\lambda) \\
\text { as } m \longrightarrow \infty,
\end{gathered}
$$

where

$$
\zeta_{k}(\lambda):=\inf _{\gamma \in \Gamma_{k}} \max _{u \in B_{k}} \Phi_{\lambda}(\gamma(u)), \quad \forall \lambda \in[1,2]
$$

with $B_{k}=\left\{u \in Y_{k}:\|u\| \leq r_{k}\right\}$ and $\Gamma_{k}:=\left\{\gamma \in C\left(B_{k}, E\right)\right.$ : $\gamma$ is odd, $\left.\left.\gamma\right|_{\partial B_{k}}=i d\right\}$.

Moreover, by the proof of Lemma 6, we have

$$
\zeta_{k}(\lambda) \in\left[\bar{\alpha}_{k}, \bar{\zeta}_{k}\right], \quad \forall k \geq k_{1}
$$

where $\bar{\zeta}_{k}:=\max _{u \in B_{k}} \Phi_{1}(u)$ and $\bar{\alpha}_{k}:=\rho_{k}^{2} / 4 \rightarrow \infty$ as $k \rightarrow \infty$ by (48).

Since the sequence $\left\{u_{m}^{k}(\lambda)\right\}_{m=1}^{\infty}$ obtained by (55) is bounded, it is clear that, for each $k \geq k_{1}$, we can choose $\lambda_{n} \rightarrow 1$ such that the sequence $\left\{u_{m}^{k}\left(\lambda_{n}\right)\right\}_{m=1}^{\infty}$ has a strong convergent subsequence.

In fact, without loss of generality, assume that

$$
\begin{gathered}
u_{m}^{k}\left(\lambda_{n}\right)^{-} \longrightarrow u_{0}^{k}\left(\lambda_{n}\right)^{-}, \quad u_{m}^{k}\left(\lambda_{n}\right)^{0} \longrightarrow u_{0}^{k}\left(\lambda_{n}\right)^{0} \\
u_{m}^{k}\left(\lambda_{n}\right)^{+} \rightarrow u_{0}^{k}\left(\lambda_{n}\right)^{+} \\
\text {as } m \longrightarrow \infty \\
u_{m}^{k}\left(\lambda_{n}\right) \rightarrow u_{0}^{k}\left(\lambda_{n}\right) \quad \text { as } m \longrightarrow \infty
\end{gathered}
$$

for some $u_{0}^{k}\left(\lambda_{n}\right)=u_{0}^{k}\left(\lambda_{n}\right)^{-}+u_{0}^{k}\left(\lambda_{n}\right)^{0}+u_{0}^{k}\left(\lambda_{n}\right)^{+} \in E=E^{-} \oplus$ $E^{0} \oplus E^{+}$since $\operatorname{dim}\left(E^{-} \oplus E^{0}\right)<\infty$.
Note that

$$
\begin{aligned}
& \Phi_{\lambda_{n}}^{\prime}\left(u_{m}^{k}\left(\lambda_{n}\right)\right) \\
& \quad=u_{m}^{k}\left(\lambda_{n}\right)^{+}-\lambda_{n}\left(u_{m}^{k}\left(\lambda_{n}\right)^{-}+\Psi^{\prime}\left(u_{m}^{k}\left(\lambda_{n}\right)\right)\right),
\end{aligned}
$$

$\forall n \in \mathbb{N}$.

That is,

$$
\begin{array}{r}
u_{m}^{k}\left(\lambda_{n}\right)^{+} \\
=\Phi_{\lambda_{n}}^{\prime}\left(u_{m}^{k}\left(\lambda_{n}\right)\right)+\lambda_{n}\left(u_{m}^{k}\left(\lambda_{n}\right)^{-}+\Psi^{\prime}\left(u_{m}^{k}\left(\lambda_{n}\right)\right)\right) \\
\forall m \in \mathbb{N} .
\end{array}
$$

In view of (55), (58), (59), and the compactness of $\Psi^{\prime}$, the right-hand side of (61) converges strongly in $E$ and hence $u_{m}^{k}\left(\lambda_{n}\right)^{+} \rightarrow u_{0}^{k}\left(\lambda_{n}\right)^{+}$in $E$. Together with (58), $\left\{u_{m}^{k}\left(\lambda_{n}\right)\right\}_{m=1}^{\infty}$ has a strong convergent subsequence in $E$.

Without loss of generality, we assume

$$
\lim _{m \rightarrow \infty} u_{m}^{k}\left(\lambda_{n}\right)=u_{n}^{k}, \quad \forall n \in \mathbb{N}, k \geq k_{1}
$$

This together with (55) and (57) yields

$$
\begin{array}{r}
\Phi_{\lambda_{n}}^{\prime}\left(u_{n}^{k}\right)=0, \quad \Phi_{\lambda_{n}}\left(u_{n}^{k}\right) \in\left[\bar{\alpha}_{k}, \bar{\zeta}_{k}\right], \\
\forall n \in \mathbb{N}, \quad k \geq k_{1} .
\end{array}
$$

Now we claim that the sequence $\left\{u_{n}^{k}\right\}_{n=1}^{\infty}$ in (63) is bounded in $E$ and possesses a strong convergent subsequence with the limit $u^{k} \in E$ for each $k \geq k_{1}$. For the sake of notational simplicity, throughout the remaining proof of Theorem 1 we always denote $u_{n}=u_{n}^{k}$.

Now we claim that $\left\{u_{n}\right\}$ is bounded in $E$. Otherwise, going to a subsequence if necessary, we can assume that $\left\|u_{n}\right\| \rightarrow \infty$ as $n \rightarrow \infty$. By (9), we have

$$
\begin{aligned}
& 2 \Phi_{\lambda_{n}}\left(u_{n}\right)-\Phi_{\lambda_{n}}^{\prime}\left(u_{n}\right) u_{n} \\
& \quad=\lambda_{n} \int_{\Omega}\left[g\left(x, u_{n}\right) u_{n}-2 G\left(x, u_{n}\right)\right] d x \\
& \quad \geq d_{3} \int_{\Omega}\left|u_{n}\right|^{\varrho} d x-d_{4} \cdot m(\Omega)
\end{aligned}
$$

which yields that

$$
\frac{\int_{\Omega}\left|u_{n}\right|^{\varrho} d x}{\left\|u_{n}\right\|} \longrightarrow 0 \text { as } n \longrightarrow \infty \text {. }
$$


Write $u_{n}=u_{n}^{-}+u_{n}^{0}+u_{n}^{+} \in E^{-} \oplus E^{0} \oplus E^{+}$. It follows from $\left(S_{1}\right)$, (24), (25), (32), and the Hölder inequality that

$$
\begin{aligned}
\Phi_{\lambda_{n}}^{\prime}\left(u_{n}\right) u_{n}^{+} \\
=\left\|u_{n}^{+}\right\|^{2}-\lambda_{n} \int_{\Omega} g\left(x, u_{n}\right) u_{n}^{+} d x \\
\geq\left\|u_{n}^{+}\right\|^{2}-2 \int_{\Omega}\left|g\left(x, u_{n}\right)\right| \cdot\left|u_{n}^{+}\right| d x \\
\geq\left\|u_{n}^{+}\right\|^{2}-d_{1} \int_{\Omega}\left|u_{n}^{+}\right| d x-d_{1} \int_{\Omega}\left|u_{n}\right|^{\nu}\left|u_{n}^{+}\right| d x \\
\geq\left\|u_{n}^{+}\right\|^{2}-d_{1}\left\|u_{n}^{+}\right\|_{1} \\
\quad-d_{1}\left(\int_{\Omega}\left(\left|u_{n}\right|^{\nu}\right)^{\varrho / v} d x\right)^{v / \varrho} \cdot\left(\int_{\Omega}\left|u_{n}^{+}\right|^{\varrho /(\varrho-v)} d x\right)^{(\varrho-v) / \varrho} \\
\geq\left\|u_{n}^{+}\right\|^{2}-c_{1}\left\|u_{n}^{+}\right\|-c_{2}\left\|u_{n}\right\|_{\varrho}^{\nu} \cdot\left\|u_{n}^{+}\right\|
\end{aligned}
$$

for any $n \in \mathbb{N}$. Here and in the sequel, we denote $c_{i}>0(i=$ $1,2, \ldots)$ for different positive constants. Since $\varrho>(2 N /(N+$ 4)) $\nu$ and $N \geq 5$, we have $v<\varrho$. So, by (65) we get

$$
\frac{\left\|u_{n}^{+}\right\|}{\left\|u_{n}\right\|} \longrightarrow 0 \quad \text { as } n \longrightarrow \infty
$$

Similarly, we have

$$
\frac{\left\|u_{n}^{-}\right\|}{\left\|u_{n}\right\|} \longrightarrow 0 \quad \text { as } n \longrightarrow \infty
$$

By $\left(S_{3}\right)$, there also exist constants $d_{6}>0$ and $d_{7}>0$ such that

$$
u g(x, u)-2 G(x, u) \geq d_{6}|u|-d_{7}, \quad \forall(x, u) \in \Omega \times \mathbb{R} .
$$

So we get

$$
\begin{aligned}
& 2 \Phi_{\lambda_{n}}\left(u_{n}\right)-\Phi_{\lambda_{n}}^{\prime}\left(u_{n}\right) u_{n} \\
& \quad=\lambda_{n} \int_{\Omega}\left[g\left(x, u_{n}\right) u_{n}-2 G\left(x, u_{n}\right)\right] d x \\
& \quad \geq d_{6} \int_{\Omega}\left|u_{n}\right| d x-d_{7} \cdot m(\Omega) \\
& \quad \geq d_{6} \int_{\Omega}\left(\left|u_{n}^{0}\right|-\left|u_{n}^{+}\right|-\left|u_{n}^{-}\right|\right) d x-d_{7} \cdot m(\Omega) \\
& \quad \geq c_{3}\left\|u_{n}^{0}\right\|-c_{4}\left(\left\|u_{n}^{-}\right\|+\left\|u_{n}^{+}\right\|\right)-c_{5}
\end{aligned}
$$

keeping in mind that $\operatorname{dim} E^{0}<\infty$ and (24). Hence, by (67) and (68), we get

$$
\frac{\left\|u_{n}^{0}\right\|}{\left\|u_{n}\right\|} \longrightarrow 0 \quad \text { as } n \longrightarrow \infty
$$

Then we arrive at

$$
1=\frac{\left\|u_{n}\right\|}{\left\|u_{n}\right\|} \leq \frac{\left\|u_{n}^{-}\right\|+\left\|u_{n}^{0}\right\|+\left\|u_{n}^{+}\right\|}{\left\|u_{n}\right\|} \longrightarrow 0 \quad \text { as } n \longrightarrow \infty
$$

which is a contradiction. Thus, $\left\{u_{n}\right\}$ is bounded in $E$. Then the proof that $\left\{u_{n}\right\}$ has a strong convergent subsequence is the same as the preceding proof of $\left\{u_{m}^{k}\left(\lambda_{n}\right)\right\}_{m=1}^{\infty}$.

Now for each $k \geq k_{1}$, by (63), the limit $u^{k}$ is just a critical point of $\Phi=\Phi_{1}$ with $\Phi\left(u^{k}\right) \in\left[\bar{\alpha}_{k}, \bar{\zeta}_{k}\right]$. Since $\bar{\alpha}_{k} \rightarrow \infty$ as $k \rightarrow \infty$ in (57), we get infinitely many nontrivial critical points of $\Phi$. Therefore, system (1) possesses infinitely many nontrivial solutions.

\section{Conflict of Interests}

The authors declare that there is no conflict of interests regarding the publication of this paper.

\section{Acknowledgments}

This study was supported by the Fundamental Research Funds for the Central Universities and the National Natural Science Foundation of China (no. 61001139).

\section{References}

[1] A. C. Lazer and P. J. McKenna, "Large-amplitude periodic oscillations in suspension bridges: some new connections with nonlinear analysis," SIAM Review, vol. 32, no. 4, pp. 537-578, 1990.

[2] A. C. Lazer and P. J. McKenna, "Global bifurcation and a theorem of Tarantello," Journal of Mathematical Analysis and Applications, vol. 181, no. 3, pp. 648-655, 1994.

[3] G. Tarantello, "A note on a semilinear elliptic problem," Differential and Integral Equations, vol. 5, no. 3, pp. 561-565, 1992.

[4] P. J. McKenna and W. Walter, "Nonlinear oscillations in a suspension bridge," Archive for Rational Mechanics and Analysis, vol. 98, no. 2, pp. 167-177, 1987.

[5] A. M. Micheletti and A. Pistoia, "Multiplicity results for a fourth-order semilinear elliptic problem," Nonlinear Analysis: Theory, Methods \& Applications, vol. 31, no. 7, pp. 895-908, 1998.

[6] A. M. Micheletti, A. Pistoia, and C. Saccon, "Three solutions of a fourth order elliptic problem via variational theorems of mixed type," Applicable Analysis, vol. 75, no. 1-2, pp. 43-59, 2000.

[7] A. M. Micheletti and C. Saccon, "Multiple nontrivial solutions for a floating beam equation via critical point theory," Journal of Differential Equations, vol. 170, no. 1, pp. 157-179, 2001.

[8] G. Xu and J. Zhang, "Existence results for some fourthorder nonlinear elliptic problems of local superlinearity and sublinearity," Journal of Mathematical Analysis and Applications, vol. 281, no. 2, pp. 633-640, 2003.

[9] Y. Pu, X. Wu, and C. Tang, "Fourth-order Navier boundary value problem with combined nonlinearities," Journal of Mathematical Analysis and Applications, vol. 398, no. 2, pp. 798-813, 2013.

[10] Y. An and R. Liu, "Existence of nontrivial solutions of an asymptotically linear fourth-order elliptic equation," Nonlinear Analysis: Theory, Methods \& Applications, vol. 68, no. 11, pp. 3325-3331, 2008.

[11] J. Liu, S. Chen, and X. Wu, "Existence and multiplicity of solutions for a class of fourth-order elliptic equations in $R^{N}$," Journal of Mathematical Analysis and Applications, vol. 395, no. 2, pp. 608-615, 2012. 
[12] X. Liu and Y. Huang, "On sign-changing solution for a fourthorder asymptotically linear elliptic problem," Nonlinear Analysis: Theory, Methods \& Applications A: Theory and Methods, vol. 72, no. 5, pp. 2271-2276, 2010.

[13] Y. Yang and J. Zhang, "Existence of solutions for some fourthorder nonlinear elliptic problems," Journal of Mathematical Analysis and Applications, vol. 351, no. 1, pp. 128-137, 2009.

[14] W. Zhang, X. Tang, and J. Zhang, "Infinitely many solutions for fourth-order elliptic equations with general potentials," Journal of Mathematical Analysis and Applications, vol. 407, no. 2, pp. 359-368, 2013.

[15] W. Zou, "Variant fountain theorems and their applications," Manuscripta Mathematica, vol. 104, no. 3, pp. 343-358, 2001.

[16] X. He and W. Zou, "Multiplicity of solutions for a class of elliptic boundary value problems," Nonlinear Analysis: Theory, Methods \& Applications, vol. 71, no. 7-8, pp. 2606-2613, 2009.

[17] Q. Zhang and C. Liu, "Multiple solutions for a class of semilinear elliptic equations with general potentials," Nonlinear Analysis: Theory, Methods \& Applications A: Theory and Methods, vol. 75, no. 14, pp. 5473-5481, 2012.

[18] H. Gu and T. An, "Infinitely many periodic solutions for subquadratic second-order Hamiltonian systems," Boundary Value Problems, vol. 2013, article 16, 2013.

[19] Q. Zhang and C. Liu, "Infinitely many periodic solutions for second order Hamiltonian systems," Journal of Differential Equations, vol. 251, no. 4-5, pp. 816-833, 2011.

[20] A. Ambrosetti and P. H. Rabinowitz, "Dual variational methods in critical point theory and applications," Journal of Functional Analysis, vol. 14, no. 4, pp. 349-381, 1973. 


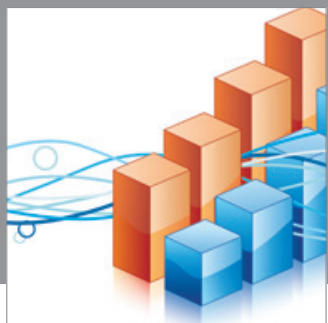

Advances in

Operations Research

mansans

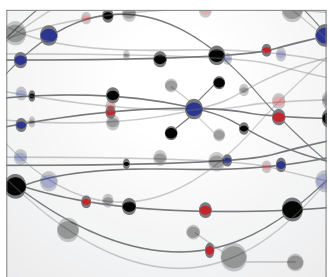

The Scientific World Journal
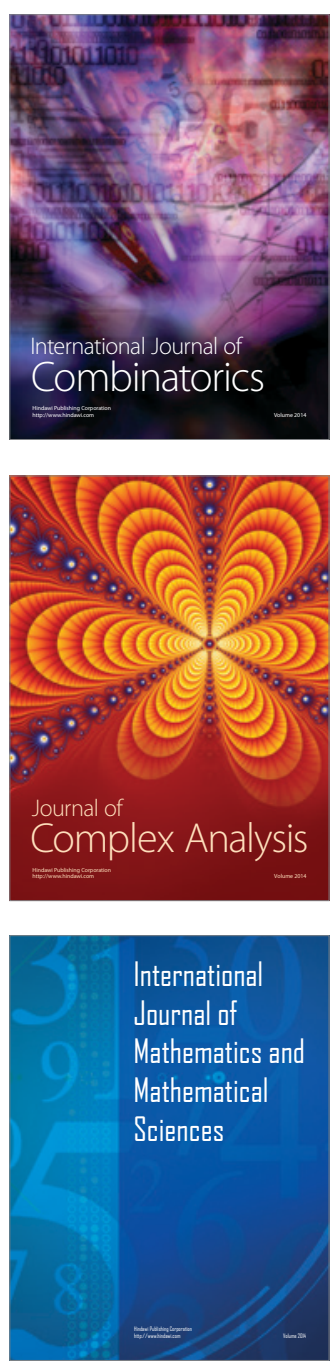
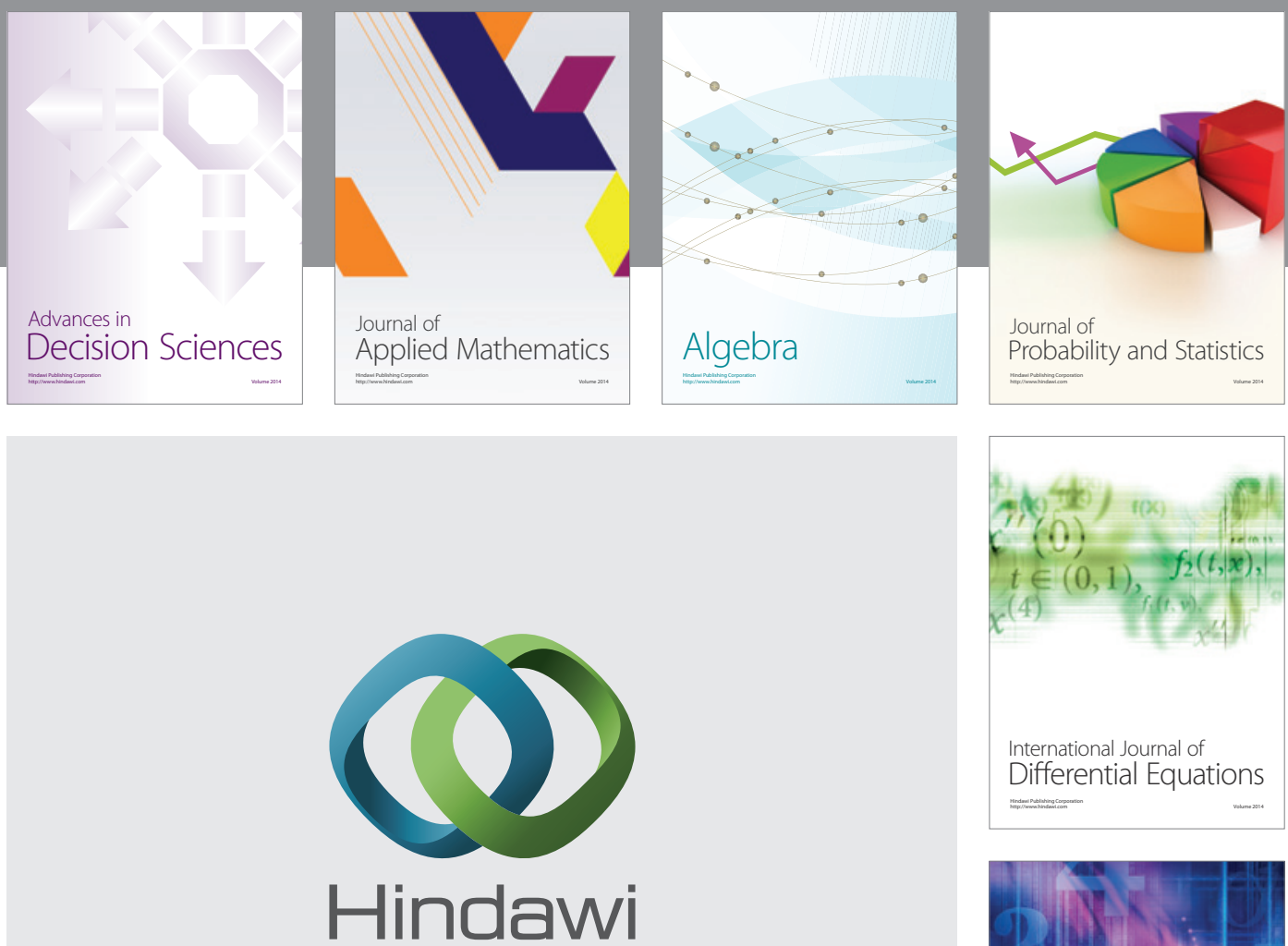

Submit your manuscripts at http://www.hindawi.com
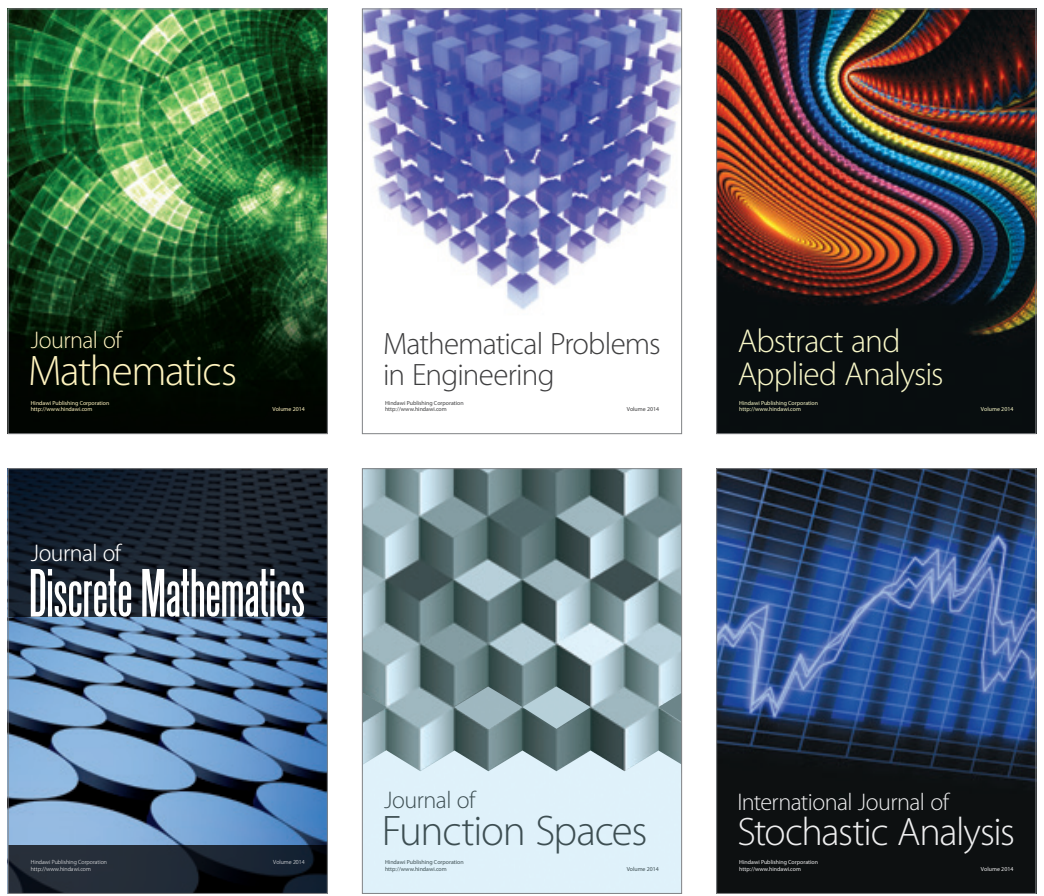

Journal of

Function Spaces

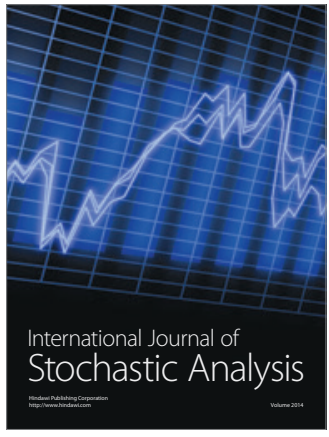

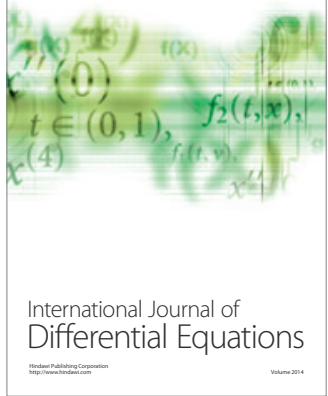
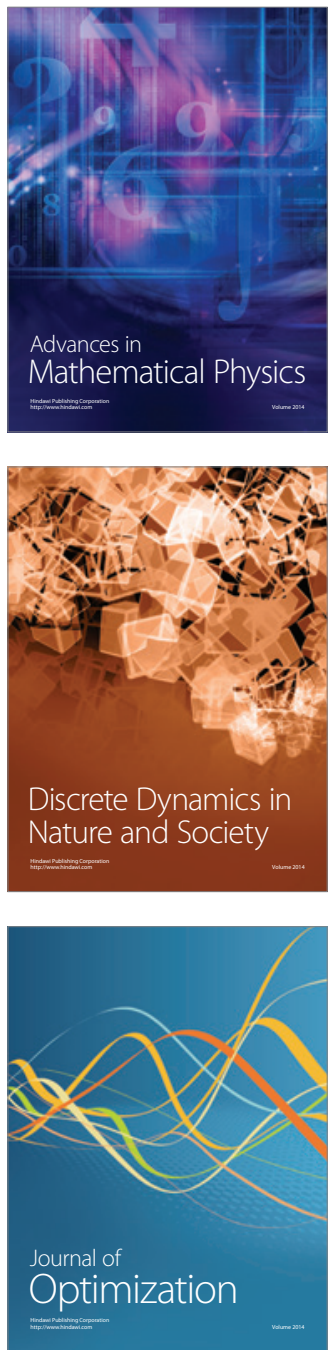